For submission to Journal of Contaminant Hydrology:

\title{
Analysis of flow behavior in fractured lithophysal reservoirs
}

\author{
Jianchun Liu, Bo Bodvarsson, and Yu-Shu Wu \\ Earth Sciences Division \\ Lawrence Berkeley National Laboratory \\ Berkeley, CA 94720, USA
}

\begin{abstract}
This study develops a mathematical model for the analysis of pressure behavior in fractured lithophysal reservoirs. The lithophysal rock is described as a tri-continuum medium, consisting of fractures, rock matrices, and cavities. In the conceptual model, fractures have homogeneous properties throughout and interact with rock matrices and cavities that have different permeabilities and porosities. Global flow occurs through the fracture network only, while rock matrices and cavities contain the majority of fluid storage and provide fluid drainage to the fractures. Interporosity flows between the triple media are described using a pseudosteady-state concept and the system is characterized by interporosity transmissivity ratios and storativity ratio of each continuum. Pressure behavior is analyzed by examining the pressure drawdown curves, the derivative plots, and the effects of the characteristic parameters. Typical pressure responses from fractures, matrices, and cavities are represented by three semilog straight lines; the transitions by two troughs below the stabilization lines in the derivative plots. The analytical solution to the proposed model is further verified using a numerical simulation. The analytical model has also been applied to a published field-buildup well test and is able to match the pressure buildup data.
\end{abstract}

Keywords: Naturally fractured reservoirs, Dual-porosity model, Triple-porosity model, Warren-Root solution, Well testing analysis, Dual-continuum medium. 


\section{Introduction}

Characterizing the behavior of naturally fractured reservoirs is important for studying the flow and transport processes in underground natural-resources recovery, waste storage, and contaminant remediation. In general, fractured rock can be considered as a multiporous medium, in which fractures and porous blocks constitute the flow system. Because of their high permeability and connectivity, fractures provide major flow channels for global fluid movement, whereas high-porosity porous blocks contain the majority of fluid storage and provide gradual fluid drainage to the fractures. Fluid flow in fractured rock is of interest in many engineering fields and has been a subject of active research for several decades. Barenblatt et al. (1960) first introduced the dual-porosity model, in which a fractured medium is represented by two completely overlapping continua, porous matrix and fractures. This double-porosity model was further developed by Warren and Root (1963) to represent the naturally fractured reservoir as an idealized system formed by identical rock-matrix blocks, separated by an orthogonally fractures. Any infinitesimal volume of the fractured formation contains a large proportion of the two constitutive media. Each point in the system is therefore assigned with two pressures, one for the fracture and the other for the matrix. Fluid exchange between the two constitutive media has been describeded by various models. A pseudosteady-state interporosity flow concept was proposed by Barenblatt et al (1960) and subsequent studies (Warren and Root, 1963; Odeh, 1965; Kazemi et al., 1969; Mavor and Cinco, 1979). Various extensions of the model have been made by assuming transient interporosity flow (de Swaan, 1976; Najurieta, 1980; Serra et al, 1983; Streltsova, 1983; Lai et al, 1983; Chen et al., 1985; Pruess and Narasimhan, 1985).

The storage capacity of naturally fractured reservoirs may vary significantly, depending on the degree of fracturing in the formation and the value of the primary porosity (Aguilera, 1980). By the definition of multiporosity theory (Aifantis, 1980), any media that exhibit finite discontinuities in the porosity field are considered to possess multiporosity properties. Single-porosity/single-permeability is in general applied to a nonfractured reservoir with uniform porosity and permeability. A fractured reservoir with relatively low matrix permeability but high storage (a "tight" reservoir) may be 
characterized by a dual-porosity/single-permeability model in which no distinction between fracture permeability and matrix permeability may be identified. A commonly accepted naturally fractured reservoir model is the dual-porosity/dual-permeability model, in which the fracture and matrix continua are distinctly different in both porosity and permeability. The high porosity/low permeability matrix and low porosity/highpermeability fracture are typical characteristics of the medium. A multiporosity/multipermeability model has been proposed in the literature (Aifantis, 1980; Abdassah and Ershaghi, 1986) to represent a severely fractured reservoir with either moderate permeability (by a triple-porosity/dual-permeability model) or high permeability (by a triple-porosity/triple-permeability model).

Honoring permeability and porosity features in a particular type of rock is crucial for a successful prediction of fluid flow and transport in the reservoir. Lithophysal rock is a hollow, bubble-like structure composed of concentric shells of finely crystalline alkali feldspar, quartz, and other minerals; it is found in certain silicic volcanic rocks, such as rhyolite and obsidian (Bates and Jackson, 1987). Lithophysal zones occur where vapor concentrates in the densely welded part of ignimbrites to form lithophysal cavities. The lithophysal cavities commonly have diameters of a few centimeters, but some are up to 1 meter across as identified in the fields, corresponding to lithophysae increases from a few percent to as much as $40 \%$ of the rock (Buesch et al., 1996).

There has been little theoretical study or modeling of flow through fractured lithophysal reservoirs in the literature. This study presents an effort of investigating flow through such rock using a tri-continuum medium concept. In the proposed triple-continuum model, fractures, rock matrices and cavities constituted the flow system. Fractures have homogeneous properties throughout and interacted with two groups of separate porous media, matrix blocks and lithophysal cavities, with different permeabilities and porosities. The geometrical configuration is that the repetitive matrix and cavity blocks are separated by a set of fractures. Each system interacts independently with other two continua. The matrix and cavity systems provide storage space but have no direct contributions to global flow and transport. The interporosity flow is proportional to the 
pressure differences between the two continua only. A pseudosteady-state flow concept was applied with pressure propagation in the fracture network. The effects of interporosity transmissivity ratios and the storativity ratio of each continuum were studied by analytically analyzing the pressure behavior of a single well with constant flow rate in an infinite lithophysal reservoir.

A numerical simulation model was constructed to examine the analytical solutions. In addition, the proposed model was also compared with a published field-buildup well-test data and successfully predicted the pressure buildup. The effects of fracture, matrix, and cavity porosities, as well as fracture and matrix permeabilities, to the pressure buildup were also analyzed. The results have both theoretical and practical significance in predicting production behaviors of carbonate reservoirs and evaluating groundwater flow and contaminant transport in such formation. The observed high flow rate in the initial stages of well flow may often lead to overestimating flow and transport processes by assuming a higher storage to exist than exists in reality. It was assuming that the high matrix block storage would continuously render the supply to the well through highly permeable fracture channels. In fact, many reservoirs that produce at high initial rates decline drastically after a short period of time because of the fluids have been stored in the fracture system. The model in this study provides more flexible tools in matching the geological variations and to avoid unrealistic predictions of reservoir flow and storage.

\section{Mathematical model}

The Warren-Root approach was used in developing the governing equations with the following basic assumptions:

1. The reservoir is of uniform thickness with impermeable lower and upper boundaries.

2. The fluid flow from the system into the wellbore is radial, and only the fractures feed the well.

3. All rock properties, such as permeability, initial porosity and compressibility, are constants in each continuum. 
4. Fluid flow is isothermal, single-phase, and slightly compressible, with constant fluid viscosity.

The governing equations describing transient fluid flow in the tri-continua system are:

$\phi_{1} C_{1} \frac{\partial p_{1}}{\partial t}-\frac{k_{1}}{\mu} \frac{1}{r} \frac{\partial}{\partial r}\left(r \frac{\partial p_{1}}{\partial r}\right)-\frac{\alpha_{12} k_{2}}{\mu}\left(p_{2}-p_{1}\right)-\frac{\alpha_{13} k_{3}}{\mu}\left(p_{3}-p_{1}\right)=0$

for the fracture continuum;

$\phi_{2} C_{2} \frac{\partial p_{2}}{\partial t}+\frac{\alpha_{12} k_{2}}{\mu}\left(p_{2}-p_{1}\right)+\frac{\alpha_{23} k_{2}}{\mu}\left(p_{2}-p_{3}\right)=0$

for the matrix continuum; and

$\phi_{3} C_{3} \frac{\partial p_{3}}{\partial t}+\frac{\alpha_{13} k_{3}}{\mu}\left(p_{3}-p_{1}\right)-\frac{\alpha_{23} k_{2}}{\mu}\left(p_{2}-p_{3}\right)=0$

for the cavity continuum. Subscripts 1, 2, and 3 are indexes for fracture, matrix, and cavity systems respectively; $p, \phi, C$, and $k$ denote pressure, initial porosity, total effective compressibility, and the permeability of each continuum, respectively; and $\alpha_{12}$, $\alpha_{13}$ and $\alpha_{23}$ are the interporosity flow shape factors, which depend on the geometry of the interporosity flow and have dimensions of reciprocal area.

The initial pressure $p_{0}$ is assumed uniform throughout the reservoir

$p_{1,2,3}(r, 0)=p_{0}$

and the same constant pressure for the outer boundary is: 


$$
p_{1,2,3}(\infty, t)=p_{0}
$$

A constant flow rate $q$ is imposed to the well with wellbore storage and skin effects ignored:

$\frac{\partial p_{1}}{\partial r}\left(r_{w}, t\right)=\frac{\mu q}{2 \pi r_{w} k_{1} h}$

where $\mu$ is fluid viscosity, $r_{w}$ well radius, and $h$ the height of the flow system.

Introducing dimensionless pressure drop $\psi_{i}$, dimensionless radial distance $\xi$, and dimensionless time $\tau$, defined as

$\psi_{i}=\frac{2 \pi k_{1} h}{\mu q}\left(p_{0}-p_{i}\right)$

$\xi=\frac{r}{r_{w}}$

and

$$
\tau=\frac{t}{\left[\mu r_{w}^{2}\left(\phi_{1} C_{1}+\phi_{2} C_{2}+\phi_{3} C_{3}\right) / k_{1}\right]}
$$

the governing equations (1)-(3), the initial conditions (4), and the boundary conditions (5)-(6) become

$\omega_{1} \frac{\partial \psi_{1}}{\partial \tau}-\frac{1}{\xi} \frac{\partial}{\partial \xi}\left(\xi \frac{\partial \psi_{1}}{\partial \xi}\right)-\lambda_{12}\left(\psi_{2}-\psi_{1}\right)-\lambda_{13}\left(\psi_{3}-\psi_{1}\right)=0$ 


$$
\begin{aligned}
& \omega_{2} \frac{\partial \psi_{2}}{\partial \tau}+\lambda_{12}\left(\psi_{2}-\psi_{1}\right)+\lambda_{23}\left(\psi_{2}-\psi_{3}\right)=0 \\
& \omega_{3} \frac{\partial \psi_{3}}{\partial \tau}+\lambda_{13}\left(\psi_{3}-\psi_{1}\right)-\lambda_{23}\left(\psi_{2}-\psi_{3}\right)=0 \\
& \psi_{1,2,3}(\xi, 0)=0 \\
& \psi_{1,2,3}(\infty, \tau)=0
\end{aligned}
$$

and

$$
\frac{\partial \psi_{1}}{\partial \xi}(1, \tau)=-1
$$

The system is now characterized by a storativity ratio for each continuum

$$
\omega_{i}=\phi_{i} C_{i} / \sum_{i=1}^{3} \phi_{i} C_{i}
$$

and the interporosity transmissivity ratio in between fracture-matrix systems:

$$
\lambda_{12}=\alpha_{12} r_{w}^{2} k_{2} / k_{1}
$$

between fracture-cavity systems:

$$
\lambda_{13}=\alpha_{13} r_{w}^{2} k_{3} / k_{1}
$$

and between matrix-cavity system: 


$$
\lambda_{23}=\alpha_{23} r_{w}^{2} k_{2} / k_{1}
$$

The mathematical model is fully defined by Equations (10)-(15). Using the Laplace transformation, the system is solved for dimensionless pressure drop $\psi_{1}$ at the wellbore as:

$\psi_{1}=\frac{1}{2}\left[\ln \tau+\ln 4-\gamma+E_{i}\left(-A_{1} \tau\right)-E_{i}\left(-B_{1} \tau\right)+E_{i}\left(-A_{2} \tau\right)-E_{i}\left(-B_{2} \tau\right)\right]$

where $\mathrm{E}_{\mathrm{i}}$ is the exponential integral function, $A_{1}, A_{2}, B_{1}$, and $B_{2}$ are constant parameters; and $\gamma=0.5772$, the Euler constant.

In the nonlithophysal case:

$A_{1}=\frac{\lambda_{12}}{\omega_{1} \omega_{2}}, B_{1}=\frac{\lambda_{12}}{\omega_{2}}, A_{2}=B_{2}=0$

and the solution becomes that of Warren-Root:

$\psi_{1}=\frac{1}{2}\left[\ln \tau+\ln 4-\gamma+E_{i}\left(-\frac{\lambda_{12}}{\omega_{1} \omega_{2}} \tau\right)-E_{i}\left(-\frac{\lambda_{12}}{\omega_{2}} \tau\right)\right]$

The detailed derivation of the solution and all constant parameters are given in the Appendix A.

Pressure behavior analysis has been enhanced by the introduction of a derivative plot (Bourdet et al., 1983; Bourdet et al., 1989). It examines the derivative of a pressure drop with respect to the natural log of time. Applying the derivative to the solution of Equation (20), we have:

$\frac{\partial \psi_{1}}{\partial \ln \tau}=\frac{1}{2}\left[1+\exp \left(-A_{1} \tau\right)+\exp \left(-A_{2} \tau\right)-\exp \left(-B_{1} \tau\right)-\exp \left(-B_{2} \tau\right)\right]$ 


\section{Analysis of pressure behavior}

\subsection{Transient pressure behavior}

Because of the three separate porosities in the fractured lithophysal reservoir, the tricontinuum system has a pressure response that may show the characteristics of combined effects. The fracture continuum, having the greatest transmissivity and being globally connected to the wellbore, responds first. The matrix and cavity continua do not flow directly into the wellbore, and therefore response at later times. The cavity continuum, having a larger transmissivity than that of the matrix, respond earlier than the matrix continuum. To demonstrate this general pressure transient behavior, a base case is set up with all the property data listed in Table 1 . The fracture-matrix transmissivity ratio in this base case is $\lambda_{12}=10^{-8}$ while fracture-cavity transmissivity ratio is $\lambda_{13}=10^{-3}$. The differences in these transmissvities lead to different response times and give rise to separate semilog straight lines on the pressure-drop curve shown in Figure 1. The pressure response from the cavity continuum in the lithophysal case occurs much earlier than that from the matrix continuum in the nonlithophysal case.

The separation between the straight lines is dependent on the storativity ratio $\omega$. In the nonlithophysal case, a clear separation exists between the fracture and the matrix line, as the fracture storativity ratio is $\omega_{1}=0.0099$ and the matrix is $\omega_{2}=0.99$. In the lithophysal case, the fracture, cavity, and matrix storativity ratios are $\omega_{1}=0.003, \omega_{2}=0.249$, and $\omega_{3}=0.748$, respectively. The cavity response can be identified by the separation between fracture and cavity lines, while the matrix response is weak with no visible line separation.

In the pressure derivative plots, the pressure behavior is characterized by a straight horizontal line during the stabilized radial flow and a trough below the straight line during the interporosity transition flow. Figure 2 shows that the trough from the fracturecavity transition in the lithophysal case arrives much earlier and is larger than that from 
the fracture-matrix transition in the nonlithophysal case. This is consistent with the pressure drop behavior exhibited in Figure 1. The matrix response in the lithophysal case comes at a later time than the cavity response and is demonstrated by a weak trough appearing after the cavity-response trough in the derivative curve.

\subsection{Effect of the cavity continuum}

We have seen that the interchange of fluids between fracture and cavity dominates wellbore pressure behavior in a fractured lithophysal reservoir. Cavities provide gradual fluid drainage to the fractures, and the pressure response from the cavity continuum is determined by fracture-cavity transmissivity ratio. A sensitivity analysis is performed by varying the fracture-cavity shape factor $\alpha_{13}$ in the base case to get three different fracturecavity transmissivity ratios, $\lambda_{13}=10^{-3}, 10^{-4}$, and $10^{-5}$. The pressure drawdown curves corresponding to the three ratios are illustrated in Figure 3. Among them, the curve of the largest value of $\lambda_{13}=10^{-3}$, representing the strongest fracture-cavity flow, shows the earliest pressure response and the least pressure drop during the transition period. Figure 4 is the derivative plot using the same data as presented in Figure 3. There are three troughs of similar shape below the stabilization line, and the arrival times of these troughs are in the order of decreasing fracture-cavity transmissivity ratios.

The fluid supplies from cavities to fractures maintain the fracture pressure and hold the pressure drawdown during the transition period. Figure 5 compares the pressure drawdown curve of fracture porosity $\phi_{\mathrm{f}}=0.0005$ with that of the base case, $\phi_{\mathrm{f}}=0.001$. With the same lithophysal volume, the transition period of smaller fracture pore volume comes earlier and lasts longer than the one with larger fracture volume. Figure 6 compares the pressure derivative plots of the same two cases. The trough of the low-fracture-porosity case arrives earlier and is larger than the one for high fracture porosity.

\subsection{Effect of the matrix continuum}

The matrix continuum in a lithophysal reservoir, because of its relative low transmissivity compared to those of fracture and cavity and its low storativity ratio compared to that of 
cavity, has less impact on pressure behavior than it has on a nonlithphysal reservoir. By varying the fracture-matrix shape factor $\alpha_{12}$ in the base case, the pressure-drawdown curves corresponding to fracture-matrix transmissivity ratios of $\lambda_{12}=10^{-8}, 10^{-6}$, and $10^{-4}$ are shown in Figure 7. As can be seen, there is little difference among three curves. This is in contrast to the nonlithophysal-case curves, plotted in the same figure for the same fracture-matrix transmissivity ratios. To study the effect of matrix-cavity interporosity flow, the matrix-cavity shape factor is increased, which gives a matrix-cavity transmissivity ratio from the base case $\lambda_{23}=10^{-6}$ to $10^{-5}$ and $10^{-4}$, respectively. Figure 8 shows the pressure drawdown and derivative curves corresponding to three ratios. There are weak troughs in the derivative plots, and the smaller matrix-cavity transmissivity ratio lead to a later trough or a later pressure response. For the highest matrix-cavity transmissivity ratio of $\lambda_{23}=10^{-4}$, the pressure response from matrix-cavity flow is so close to the response from fracture-cavity flow, that the two troughs are connected to each other. The overall differences among the three cases are insignificant and can hardly be identified in the pressure-drawdown curves.

For a lithophysal reservoir of a small cavity volume, the matrix effect could become relatively strong as the reservoir acts more like a nonlithophysal reservoir. Figure 9 shows a case with a cavity porosity of $\phi_{\mathrm{c}}=0.05$ and a matrix porosity of $\phi_{\mathrm{m}}=0.3$. Compared to the base case of strong lithophysal $\left(\phi_{\mathrm{c}}=0.3, \phi_{\mathrm{m}}=0.1\right)$, the weak lithophysal case has clear separations between three semilog straight lines corresponding to fracture, matrix, and cavity-pressure responses. In the derivative plot shown in Figure 10, the weak lithophysal case also has two distinct troughs, one from the cavity response and one from the matrix response, with the matrix trough arriving at a later time. The storativity ratios in the weak lithophysal case are $\omega_{2}=0.855$ for the matrix and $\omega_{3}=0.143$ for the cavity while in the base case of strong lithophysal are $\omega_{2}=0.249$ and $\omega_{3}=0.748$, respectively.

\subsection{Comparison with Numerical Simulation}

A numerical simulation was performed to examine the analytical solutions. The radial flow is considered single-phase, and slightly compressible in an infinite-acting reservoir. 
The flow occurs into a fully penetrating well from a horizontal, uniform, and fractured lithophysal zone. Wellbore storage and skin effects are ignored. The wellbore radius, formation height, porosity, permeability, compressibility and fluid property data are the same as those used for the analytical solution. The reservoir was simulated using the TOUGH2 code (Pruess et al., 1991) by a one-dimensional, radially symmetrical, infiniteacting reservoir with an outer boundary of 10,000 meters. The radial distance was subdivided into intervals as a primary grid. A triple-porosity mesh, consisting of a threedimensional fracture network with cubic matrix and cavity blocks, was generated from the primary grid. For a given interporosity flow shapefactor, $\alpha$, the matrix and cavity block sizes are assumed uniform and determined using the Warren-Root pseudo steadystate model. A fully penetrating well is represented by a well element having a specified constant flow rate. Simulations are performed using the base case parameters (Table 1) and three fracture-cavity transmissivity ratios, $\lambda_{13}=10^{-3}, 10^{-4}$, and $10^{-5}$. Good agreements between the analytical solution and simulation results are obtained, as shown in Figure 11.

\subsection{Application to analysis of field data}

Crawford et al. (1976) presented some field pressure buildup data (Table 2) that demonstrated the pressure response of a naturally fractured reservoir and supported the existence of the triple-porosity system. The test well was shut in after about 10 hours and pressure-buildup data were recorded with high-precision pressure gauges from a well, which produced from a two-layer reservoir. The well is commingled where most of the deliverability is coming from the bottom few feet of the wellbore and a small percentage of deliverability is from the zone of undefined thickness more than $400 \mathrm{ft}$ above. The lower zone is believed to be a naturally fractured formation. Because there are two regions that have different reservoir properties (lower and upper zones) interacting, this system follows the triple-porosity model. The buildup curves appear to have straight-line segments with transition regions between them. The analytical model developed in this study can match the pressure response quite well (Figure 12) with the model parameters given in Table 3. Fracture porosity and matrix permeability were estimated using the 
fracture permeability, matrix porosity, fracture-matrix storativity ratio, and fracturematrix transmissivity ratio given by Crawford et al. (1976). The shape factors were evaluated using the same definition as that given by Abdassah and Ershaghi (1986). Cavity porosity and permeability were obtained by the best-matched pressure-buildup curves. The storativity ratio of fracture, matrix, and cavity are $0.006,0.9$, and 0.085 , respectively. The transmissivity ratio between fracture and matrix is $4.43 \times 10^{-7}$; between fracture and cavity it is $2.92 \times 10^{-6}$. Pressure buildup behavior, and the effects of fracture, matrix, and cavity porosities, as well as fracture and matrix permeabilities were shown in Figures 13-17. The cavity volume has more impact on the timing of the transition period than the matrix volume. Fracture porosity and permeability affect the early pressure build up while matrix permeability dominates at later time.

\section{Conclusions}

We have developed a new mathematical model for analyzing transient pressure behavior in fractured lithophysal reservoirs. The model is based on single-phase flow from or into a single well, with constant rate in an infinite reservoir. The lithophysal rock is described as a tri-continuum medium, consisting of fractures, rock matrices and cavities. Fractures have homogeneous properties throughout and interact directly with rock matrices and cavities that have different permeabilities and porosities. Global flow or transient pressure propagates in the fracture network, while rock matrices and cavities contain the majority of fluid storage and provide gradual fluid drainage to the fractures.

The pseudosteady-state concept was applied to interporosity flows and pressure behavior was analyzed by examining the effects of interporosity transmissivity ratios and the storativity ratio of each continuum. Pressure responses from fractures, matrices and cavities are found to be represented by three semilog straight lines with the responding times determined by the transmissivity ratios. Separations between the lines and the length of the transition period are determined by the storativity ratios. Two troughs appear and correspond to two transition periods in the pressure-derivative plots. Pressure responses from cavities are much stronger than those from matrices. 
A numerical simulation was conducted to verify the analytical solutions. The simulation model used three sets of orthogonal fractures separated by cubic matrix and cavity blocks. The simulation results were found to be in excellent agreement with the analytical solutions. Furthermore, the analytical model was applied to a published field-buildup well test and was able to match the pressure buildup data.

The model developed in this study has both theoretical and practical significance in predicting production behaviors of carbonate reservoirs and evaluating groundwater flow and contaminant transport. Many fractured reservoirs have very high initial production rates, but decline drastically in production after a short period of time, because the early produced fluids come primarily from the fracture system of very small volume. The observed high flow rate may often lead to overestimating flow and transport processes by assuming a higher storage and the high storage would continuously render the supply to the well through highly permeable fracture channels. The results from this study provide an explanation of such a phenomenon and a more flexible and useful tools in matching the geological variations and to avoid unrealistic predictions of reservoir flow and storage. The dual-porosity model is shown to be a special case of the present model. For practical ranges of well testing duration, the behavior of the two most contributing types of fluid storage continua may be analyzed with the proposed model. The concept, however, can be generalized to include multiporosity systems. Note that the analytical solution was derived using the pseudosteady-state interporosity flow assumption. This was because of the mathematica difficulty in treatment of transient interporosity flow. This limits the transient effect to be of only short duration (Gringarten, 1984). The improvement can be made by simplify the geometry of storage matrix blocks, depending on the geology of the reservoirs.

\section{Notation}

a parameter in the pressure solution

$\mathrm{A}_{1}, \mathrm{~A}_{2} \quad$ parameter in the pressure solution 


\begin{tabular}{ll}
$\mathrm{b}$ & parameter in the pressure solution \\
$\mathrm{B}_{1}, \mathrm{~B}_{2}$ & parameter in the pressure solution \\
$\mathrm{C}$ & ${\text { compressibility, } \mathrm{Pa}^{-1}}$ \\
$\mathrm{~h}$ & formation height, $\mathrm{m}$ \\
$\mathrm{k}$ & permeability, $\mathrm{m}^{2}$ \\
$\mathrm{~K}_{0}$ & modified Bessel function of the second kind, zero order \\
$\mathrm{K}_{1}$ & modified Bessel function of the second kind, first order \\
$\mathrm{p}$ & pressure, Pa \\
$\mathrm{p}_{0}$ & initial and outer boundary pressure, Pa \\
$\mathrm{q}$ & well flow rate, $\mathrm{m}^{3} / \mathrm{s}$ \\
$\mathrm{r}$ & radial distance, $\mathrm{m}$ \\
$\mathrm{r}_{\mathrm{w}}$ & wellbore radius, $\mathrm{m}$ \\
$\mathrm{s}$ & Laplace transformation variable, $\mathrm{s}$ \\
$\mathrm{t}$ & time, $\mathrm{s}$ \\
$\mathrm{u}$ & Laplace transformation function, $\mathrm{s}$ \\
$\alpha$ & interporosity shape factor, $\mathrm{m}^{-2}$ \\
$\phi$ & initial or reference porosity \\
$\gamma$ & Euler constant \\
$\lambda$ & interporosity transmissivity ratio \\
$\mu$ & fluid viscosity, sPa \\
$\tau$ & dimensionless time \\
$\xi$ & storativity ratio \\
\hline & dimensionless radial distance \\
\hline & dimensionless pressure \\
\hline &
\end{tabular}

Subscripts

1 fracture continuum

2 matrix continuum

3 cavity continuum 


\section{Acknowledgment}

We would like to thank Lehua Pan and Dan Hawkes for their review of this paper. We are also indebted to Jil Geller and James E. Hoseworth for their insightful and constructive comments during the JHC review. This work was supported by the Director, Office of Civilian Radioactive Waste Management, U. S. Department of Energy, through Memorandum Purchase Order EA9013MC5X between TRW Environmental Safety Systems Inc. and the Ernest Orlando Lawrence Berkeley National Laboratory. The support is provided to Lawrence Berkeley National Laboratory through the U. S. Department of Energy Contract No. DE-AC03-76SF00098.

\section{References}

Abdassah, D. and Ershaghis I., 1986. Triple-porosity system for representing naturally fractured reservoirs. SPE Form. Eval., 1, 113-127.

Aifantis, E.C., 1980. On the problem of diffusion in solids. Acta Mech., 37, 265-296.

Aguilera, R., 1980. Naturally Fractured Reservoirs, Petroleum, Tulsa, Oklahoma.

Barenblatt, G.E., Zheltov, I.P., and Kochina, I.N., 1960. Basic concepts in the theory of homogeneous liquids in fissured rocks. J. Appl. Math. Mech. 24, 1286-1303.

Bates, R.L., and Jakson, J.L., 1987. Glossary of Geology: Alexandia, Virginia, American Geological Institute 788.

Bourdet, D., Whittle, T.M., Douglas, A.A., and Pirard, Y.M., 1983. A new set of type curves simplifies well test analysis. World Oil 95-106.

Bourdet, D., Ayoub, J.A., and Pirard, Y.M., 1989. Use of the pressure derivative in well test interpretation. SPE Form. Eval., 293-302.

Buesch, D.C., Spengler, R.W., Moyer, T.C., and Geslin, J.K., 1996. Proposed stratigraphic nomenclature and macroscope identification of lithostratigraphic units of the paintbrust group exposed at Yucca Mountain, Nevada. U.S. Geological Survey Open-File Report 94-469. U.S. Geological Survey, Denver, Colorado.

Chen, C.C., Serra, K.,Reynolds, A.C., and Raghavan, R., 1985. Pressure transient analysis methods for bounded naturally fractured reservoirs. Soc. Pet. Eng. J. 25, 451-464.

Crawford, G.E., Hagedorn, A.R., and Pierce, A.E., 1976. Analysis of pressure buildup test in a naturally fractured reservoir. J. Pet. Tech. 1295-1300. 
de Swaan, A.O., 1976. Analytical solution for determining naturally fractured reservoir properties by well testing. Soc. Pet. Eng. J. 117-122.

Gringarten, A.C., 1984. Interpretation of tests in fissured and multilayered reservoirs with double-porosity behavior: theory and practice. J. Pet. Tech. 549-64.

Kazemi, H., Seth, M.S., and Thomas, G.W., 1969. The interpretation of interference tests in naturally fractured reservoirs with uniform fracture distribution. Soc. Pet. Eng. J. 463-72; Trans. AIME, 246.

Lai, C.H., Bodvarsson, G.S., Tsang, C.F., and Witherspoon, P.A., 1983. A new model for well test data analysis for naturally fractured reservoirs. Paper SPE 11688 presented at the 1983 SPE California Regional Meeting, Ventura, March 23-25.

Mavor, M.J. and Cinco, H., 1979. Transient pressure behavior of naturally fractured reservoirs. Paper SPE 7977 presented at the 1979 SPE California Regional Meeting, Ventura, April 18-20.

Najurieta, H.L., 1980. A theory for pressure transient analysis in naturally fractured reservoirs. J. Pet. Tech. 117-122.

Odeh, A.S., 1965. Unsteady-state behavior of naturally fractured reservoirs. Soc. Pet. Eng. J. 60-66; Trans. AIME, 234.

Pruess, K. and Narasimhan, T.N., 1985. A practical method for modeling fluid and heat flow in fractured porous media. Soc. Pet. Eng. J. 25, 14-26.

Pruess, K., 1991. TOUGH2-A general purpose numerical simulator for multiphase fluid and heat flow. Report LBL-29400. Lawrence Berkeley National Laboratory, Berkeley, California.

Serra, K., Reynolds, A.C., and Raghavan, R., 1983. New pressure transient analysis methods for naturally fractured reservoirs. J. Pet. Tech. 2271-83.

Streltsova T.D., 1983. Well pressure behavior of a naturally fractured reservoir. Soc. Pet. Eng. J. 769-80.

Warren, J. E. and Root, P.J., 1963. Behavior of naturally fractured reservoirs. Soc. Pet. Eng. J. 235-55; Trans. AIME, 228. 


\section{Appendix A: Derivation of Analytical Solutions}

The mathematical model of the tri-continua system is fully defined by the dimensionless governing equations

$\omega_{1} \frac{\partial \psi_{1}}{\partial \tau}-\frac{1}{\xi} \frac{\partial}{\partial \xi}\left(\xi \frac{\partial \psi_{1}}{\partial \xi}\right)-\lambda_{12}\left(\psi_{2}-\psi_{1}\right)-\lambda_{13}\left(\psi_{3}-\psi_{1}\right)=0$

$\omega_{2} \frac{\partial \psi_{2}}{\partial \tau}+\lambda_{12}\left(\psi_{2}-\psi_{1}\right)+\lambda_{23}\left(\psi_{2}-\psi_{3}\right)=0$

$\omega_{3} \frac{\partial \psi_{3}}{\partial \tau}+\lambda_{13}\left(\psi_{3}-\psi_{1}\right)-\lambda_{23}\left(\psi_{2}-\psi_{3}\right)=0$

with initial condition

$\psi_{1,2,3}(\xi, 0)=0$

and boundary conditions

$\psi_{1,2,3}(\infty, \tau)=0$

$\frac{\partial \psi_{1}}{\partial \xi}(1, \tau)=-1$

Applying Laplace transformation to Equations (A1) through (A6) yileds:

$$
\begin{aligned}
& \omega_{1} s u_{1}-\frac{1}{d} \frac{}{\xi}\left(\xi \frac{d u_{1}}{d \xi}\right)-\lambda_{12}\left(u_{2}-u_{1}\right)-\lambda_{13}\left(u_{3}-u_{1}\right)=0 \\
& \omega_{2} s u_{2}+\lambda_{12}\left(u_{2}-u_{1}\right)+\lambda_{23}\left(u_{2}-u_{3}\right)=0 \\
& \omega_{3} s u_{3}+\lambda_{13}\left(u_{3}-u_{1}\right)-\lambda_{23}\left(u_{2}-u_{3}\right)=0 \\
& u_{1}(\infty, s)=0
\end{aligned}
$$

and

$$
\frac{d u_{1}}{d \xi}(1, s)=-1 / s
$$


where $u_{1}, u_{2}$ and $u_{3}$ are transformed functions of $\psi_{1}, \psi_{2}$ and $\psi_{3}$ in the Laplace domain, and $s$ is the transformation variable.

Substituting the matrix and cavity equations into the fracture equation, we have

$\frac{1}{\xi} \frac{d}{d \xi}\left(\xi \frac{d u_{1}}{d \xi}\right)-s f(s) u_{1}=0$

where

$$
f(s)=\omega_{1}+\frac{\left(\lambda_{12}+\lambda_{13}\right) s+\frac{1-\omega_{1}}{\omega_{2} \omega_{3}}\left[\lambda_{12} \lambda_{13}+\left(\lambda_{12}+\lambda_{13}\right) \lambda_{23}\right]}{s^{2}+\left[\frac{\lambda_{12}}{\omega_{2}}+\frac{\lambda_{13}}{\omega_{3}}+\left(\frac{1}{\omega_{2}}+\frac{1}{\omega_{3}}\right) \lambda_{23}\right] s+\frac{\lambda_{12} \lambda_{13}+\left(\lambda_{12}+\lambda_{13}\right) \lambda_{23}}{\omega_{2} \omega_{3}}}
$$

The solution subject to boundary conditions is

$u_{1}=\frac{K_{0}(\sqrt{s f(s)})}{s \sqrt{s f(s)} K_{1}(\sqrt{s f(s)})}$

where $K_{0}$ and $K_{1}$ are Bessel functions.

Considering the approximations $K_{0}(v)=-(\gamma+\ln v-\ln 2)$ and $K_{1}(v)=1 / v$ for large time $(\tau>100)$, we can obtain the dimensionless pressure drop $\psi_{1}$ after inverting $u_{1}$ to physical domain

$\psi_{1}=\frac{1}{2}\left[\ln \tau+\ln 4-\gamma+E_{i}\left(-A_{1} \tau\right)-E_{i}\left(-B_{1} \tau\right)+E_{i}\left(-A_{2} \tau\right)-E_{i}\left(-B_{2} \tau\right)\right]$

where $\mathrm{E}_{\mathrm{i}}$ is the exponential integral function, $A_{1}, A_{2}, B_{1}$ and $B_{2}$ are given by:

$$
\begin{aligned}
& A_{1}=a+\frac{\lambda_{12}+\lambda_{13}}{2 \omega_{1}}+\left[\left(a+\frac{\lambda_{12}+\lambda_{13}}{2 \omega_{1}}\right)^{2}-\frac{b}{\omega_{1}}\right]^{1 / 2} \\
& A_{2}=a+\frac{\lambda_{12}+\lambda_{13}}{2 \omega_{1}}-\left[\left(a+\frac{\lambda_{12}+\lambda_{13}}{2 \omega_{1}}\right)^{2}-\frac{b}{\omega_{1}}\right]^{1 / 2} \\
& B_{1}=a+\left(a^{2}-b\right)^{1 / 2}
\end{aligned}
$$

and 
$B_{2}=a-\left(a^{2}-b\right)^{1 / 2}$

with

$a=\frac{1}{2}\left[\frac{\lambda_{12}}{\omega_{2}}+\frac{\lambda_{13}}{\omega_{3}}+\left(\frac{1}{\omega_{2}}+\frac{1}{\omega_{3}}\right) \lambda_{23}\right]$

and

$b=\frac{\lambda_{12} \lambda_{13}+\left(\lambda_{12}+\lambda_{13}\right) \lambda_{23}}{\omega_{2} \omega_{3}}$

and $\gamma=0.5772$, the Euler constant.

In the case of nonlithophysal:

$a=\frac{\lambda_{12}}{\omega_{2}}, b=0$,

$A_{1}=\frac{\lambda_{12}}{\omega_{1} \omega_{2}}, B_{1}=\frac{\lambda_{12}}{\omega_{2}}, A_{2}=B_{2}=0$

and the solution becomes that of Warren-Root:

$\psi_{1}=\frac{1}{2}\left[\ln \tau+\ln 4-\gamma+E_{i}\left(-\frac{\lambda_{12}}{\omega_{1} \omega_{2}} \tau\right)-E_{i}\left(-\frac{\lambda_{12}}{\omega_{2}} \tau\right)\right]$ 
Table 1. Base-case data

Fluid viscosity

Wellbore radius

Fracture porosity

Matrix porosity

Cavity porosity

Fracture permeability

Matrix permeability

Cavity permeability

Fracture compressibility

Matrix compressibility

Cavity compressibility

Fracture-matrix shape factor

Fracture-cavity shape factor

Matrix-cavity shape factor

Flow rate

Formation thickness
$10^{-3} \mathrm{sPa}$

$0.1 \mathrm{~m}$

0.001

0.1

0.3

$10^{-12} \mathrm{~m}^{2}$

$10^{-16} \mathrm{~m}^{2}$

$10^{-10} \mathrm{~m}^{2}$

$10^{-9} \mathrm{~Pa}^{-1}$

$10^{-9} \mathrm{~Pa}^{-1}$

$10^{-9} \mathrm{~Pa}^{-1}$

$0.01 \mathrm{~m}^{-2}$

$0.1 \mathrm{~m}^{-2}$

$1 \mathrm{~m}^{-2}$

$10^{-3} \mathrm{~m}^{3} / \mathrm{s}$

$10 \mathrm{~m}$ 
Table 2. Field buildup well test data (Crawford et al. 1976)

Fluid viscosity

Wellbore radius

Matrix porosity

Compressibility

Flow rate

Formation thickness

Volume factor

Horner time

Initial pressure

$\begin{array}{cc}\text { Time (hours) } & \text { Pressure (psi) } \\ & \\ 0.101 & 3608.7 \\ 0.134 & 3610.9 \\ 0.168 & 3611.8 \\ 0.201 & 3613.0 \\ 0.235 & 3613.9 \\ 0.369 & 3616.6 \\ 0.603 & 3617.3 \\ 0.671 & 3618.8 \\ 0.839 & 3619.7 \\ 1.006 & 3620.1 \\ 1.174 & 3620.5 \\ 1.342 & 3620.7 \\ 1.510 & 3620.7 \\ 1.677 & 3621.3 \\ 1.879 & 3621.7 \\ 2.080 & 3621.9 \\ 2.281 & 3621.9 \\ 2.482 & 3621.9 \\ 2.684 & 3621.9 \\ 2.885 & 3622.3 \\ 3.086 & 3622.3 \\ 3.288 & 3622.3\end{array}$

$$
\begin{aligned}
& 4.2 \mathrm{cp} \\
& 0.25 \mathrm{ft} \\
& 0.15 \\
& 10^{-5} \mathrm{psi}^{-1} \\
& 1292 \mathrm{Standard} \mathrm{Barrel} / \text { Day }_{175 \mathrm{ft}} \\
& 1.23 \mathrm{Reservoir} \text { Barrel/Standard Barrel } \\
& 10.87 \text { hours } \\
& 3628 \mathrm{psi}
\end{aligned}
$$

Time (hours) Pressure (psi)

$\begin{array}{cc}3.489 & 3622.4 \\ 3.690 & 3622.4 \\ 3.891 & 3622.3 \\ 4.227 & 3622.5 \\ 4.562 & 3622.5 \\ 4.898 & 3622.9 \\ 5.233 & 3622.6 \\ 5.569 & 3622.6 \\ 5.904 & 3622.7 \\ 6.240 & 3622.6 \\ 6.575 & 3622.9 \\ 6.877 & 3623.0 \\ 7.213 & 3622.8 \\ 7.548 & 3623.4 \\ 8.219 & 3623.4 \\ 9.158 & 3623.3 \\ 9.796 & 3623.3 \\ 9.896 & 3623.6 \\ 9.997 & 3623.8 \\ 10.098 & 3623.7 \\ 10.198 & 3623.3 \\ 10.467 & 3623.5\end{array}$


Table 3. Model parameters for the field-buildup test case

Fluid viscosity

Wellbore radius

Fracture porosity

Matrix porosity

Cavity porosity

Fracture permeability

Matrix permeability

Cavity permeability

Fracture compressibility

Matrix compressibility

Cavity compressibility

Fracture-matrix shape factor

Fracture-cavity shape factor

Matrix-cavity shape factor

Flow rate

Formation thickness

Volume factor

Horner time

Initial pressure
$4.2 \mathrm{cp}$

$0.25 \mathrm{ft}$

0.001

0.15

0.014

$350 \mathrm{md}$

$76 \mathrm{md}$

$500 \mathrm{md}$

$10^{-5} \mathrm{psi}^{-1}$

$10^{-5} \mathrm{psi}^{-1}$

$10^{-5} \mathrm{psi}^{-1}$

$3.26 \times 10^{-5} \mathrm{ft}^{-2}$

$3.26 \times 10^{-5} \mathrm{ft}^{-2}$

0

1292 Standard Barrel/Day

$175 \mathrm{ft}$

1.23 Reservoir Barrel/Standard Barrel 10.87 hours

3628 psi

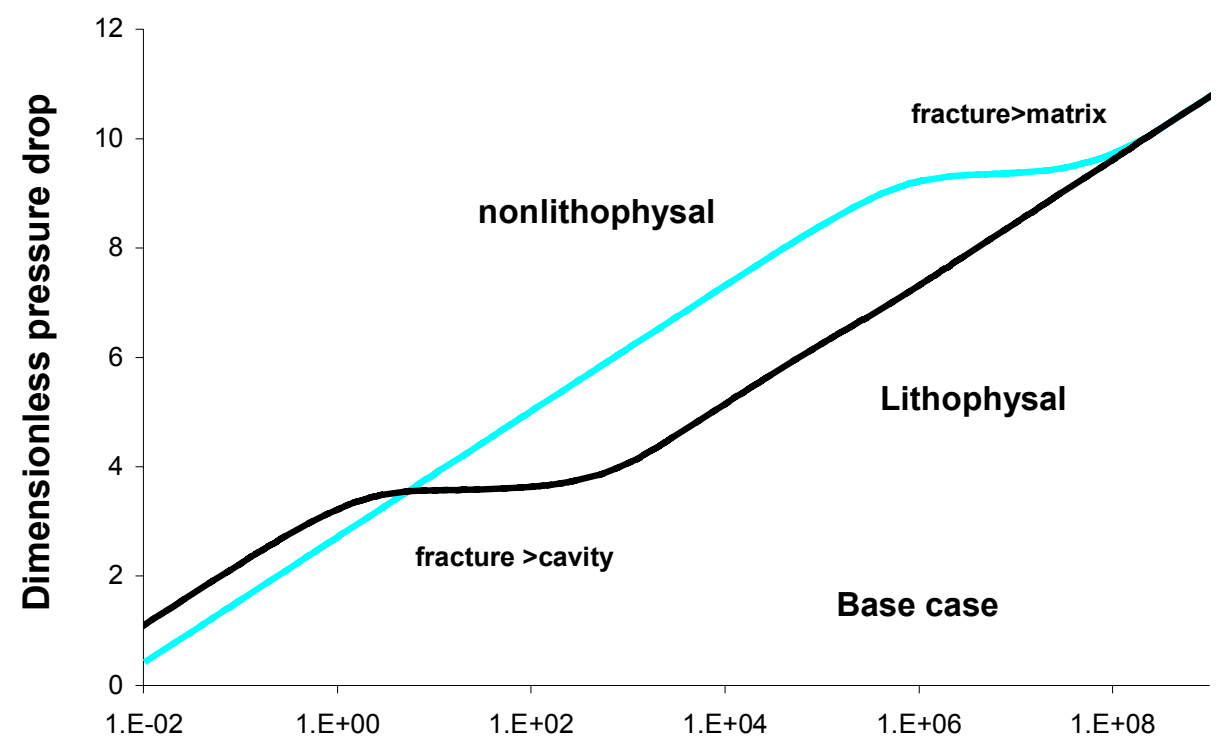

Dimensionless time

Fig. 1 Pressure drawdown curve of the base case. 


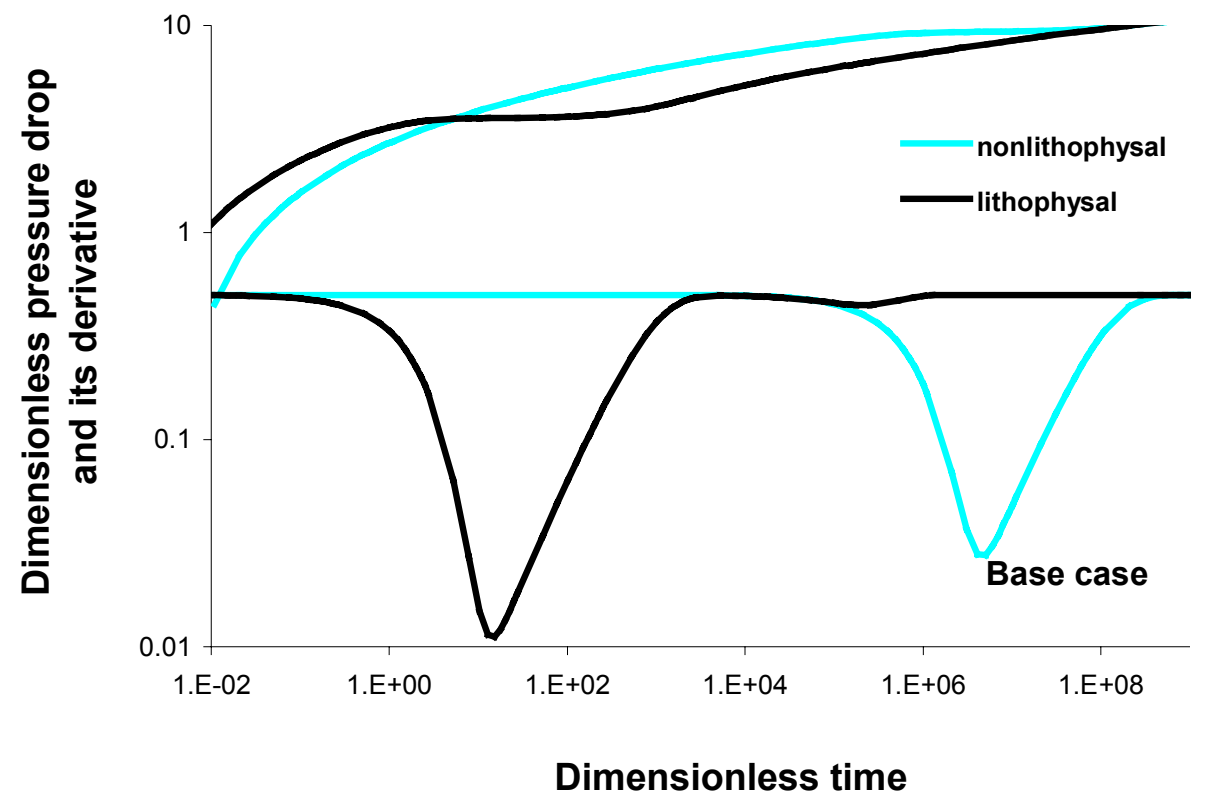

Fig. 2. Pressure drawdown curve and derivative plots of the base case.

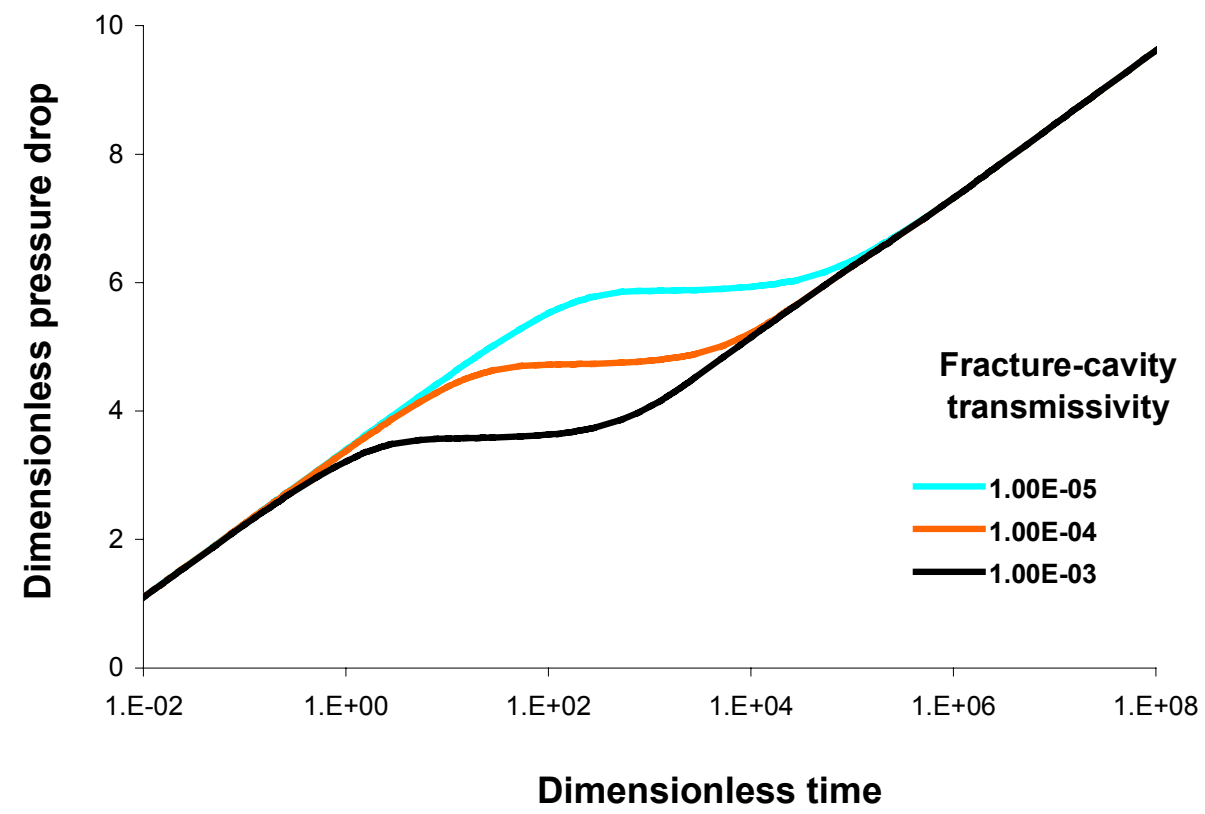

Fig. 3. Sensitivity of fracture-cavity transmissivity ratio displayed in pressure drawdown curve. 


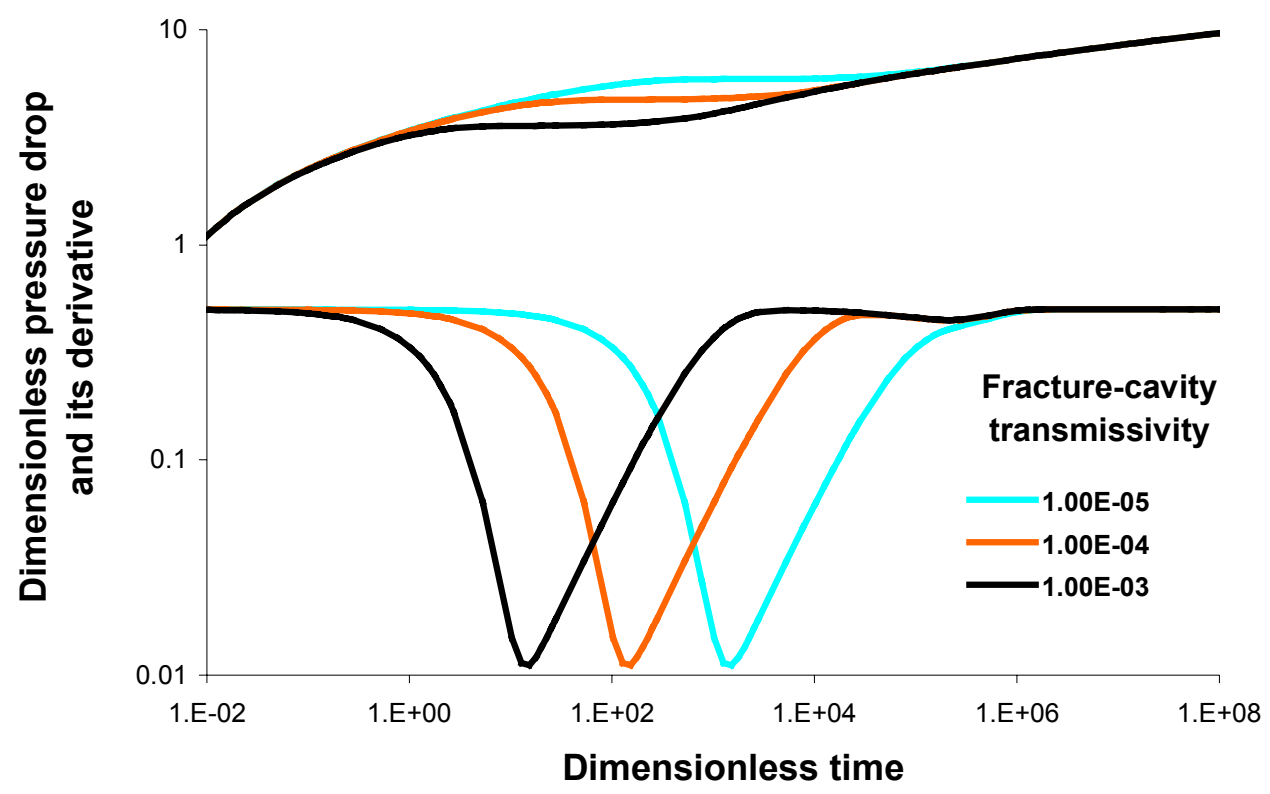

Fig. 4. Sensitivity of fracture-cavity transmissivity ratio displayed in pressure derivative plot.

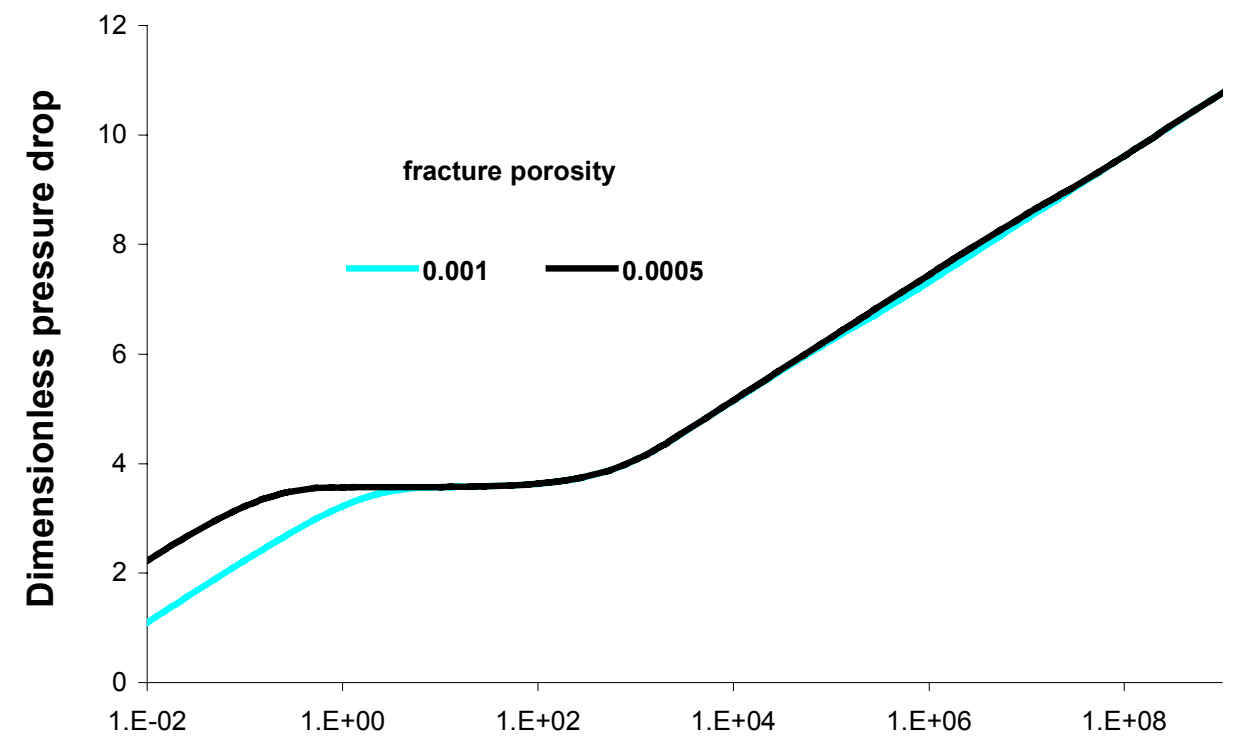

Dimensionless time

Fig. 5. Sensitivity of fracture volume displayed in pressure drawdown curve. 


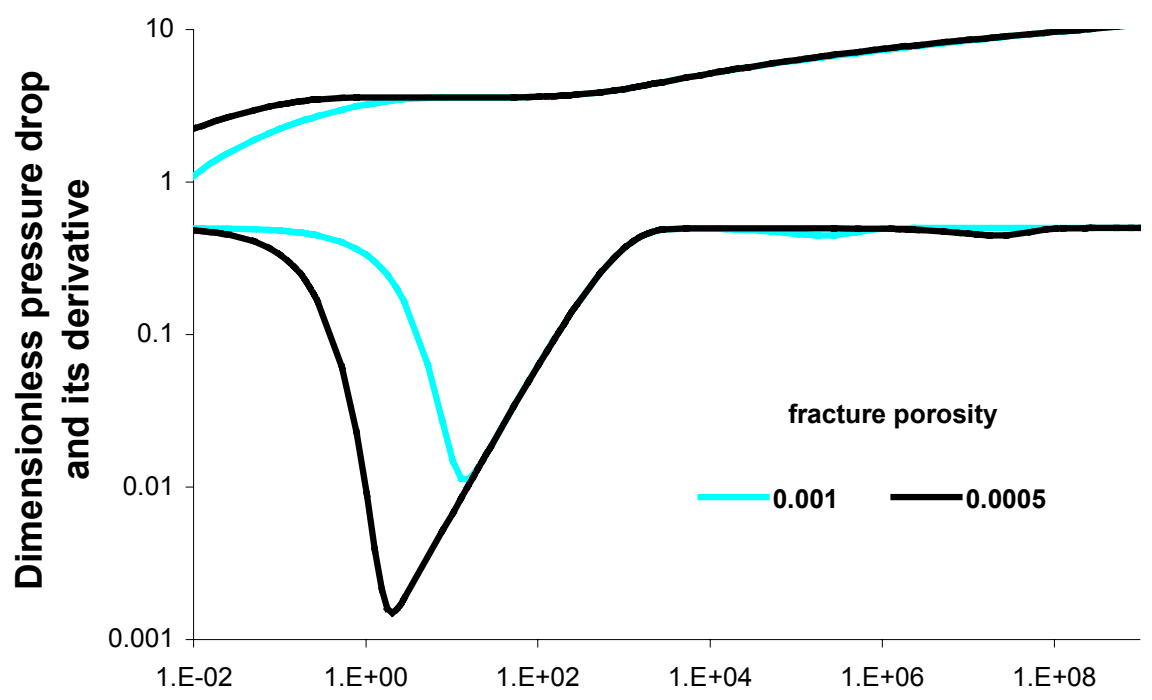

Dimensionless time

Fig. 6. Sensitivity of fracture volume displayed in pressure derivative plot.

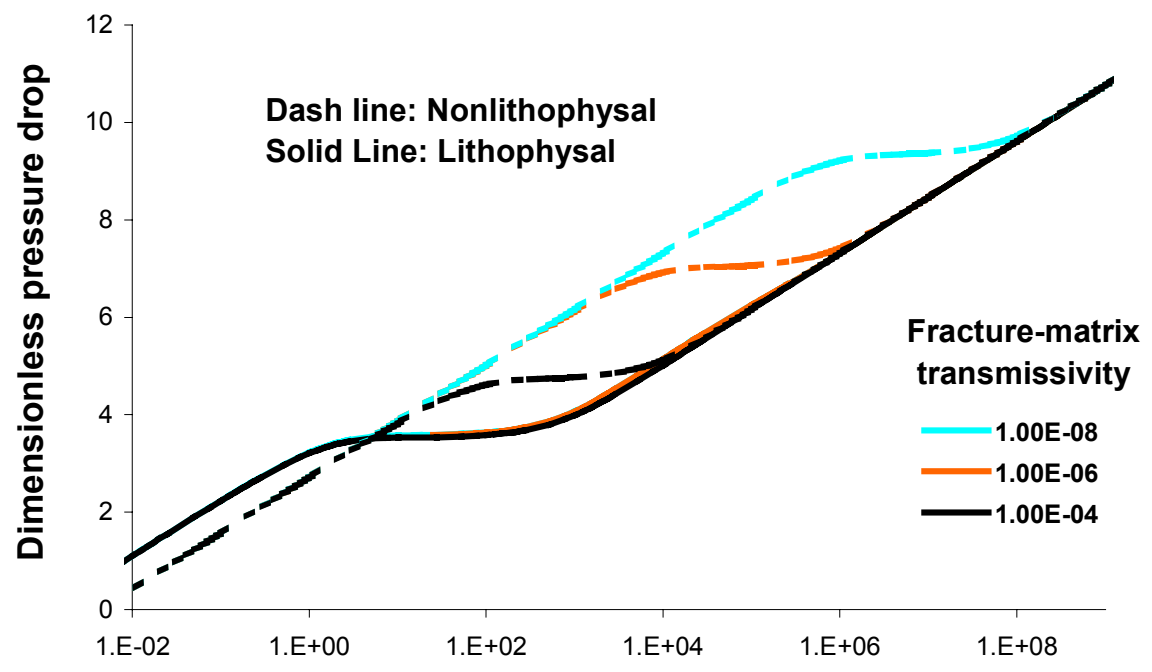

\section{Dimensionless time}

Fig. 7. Sensitivity of fracture-matrix transmissivity ratio. 


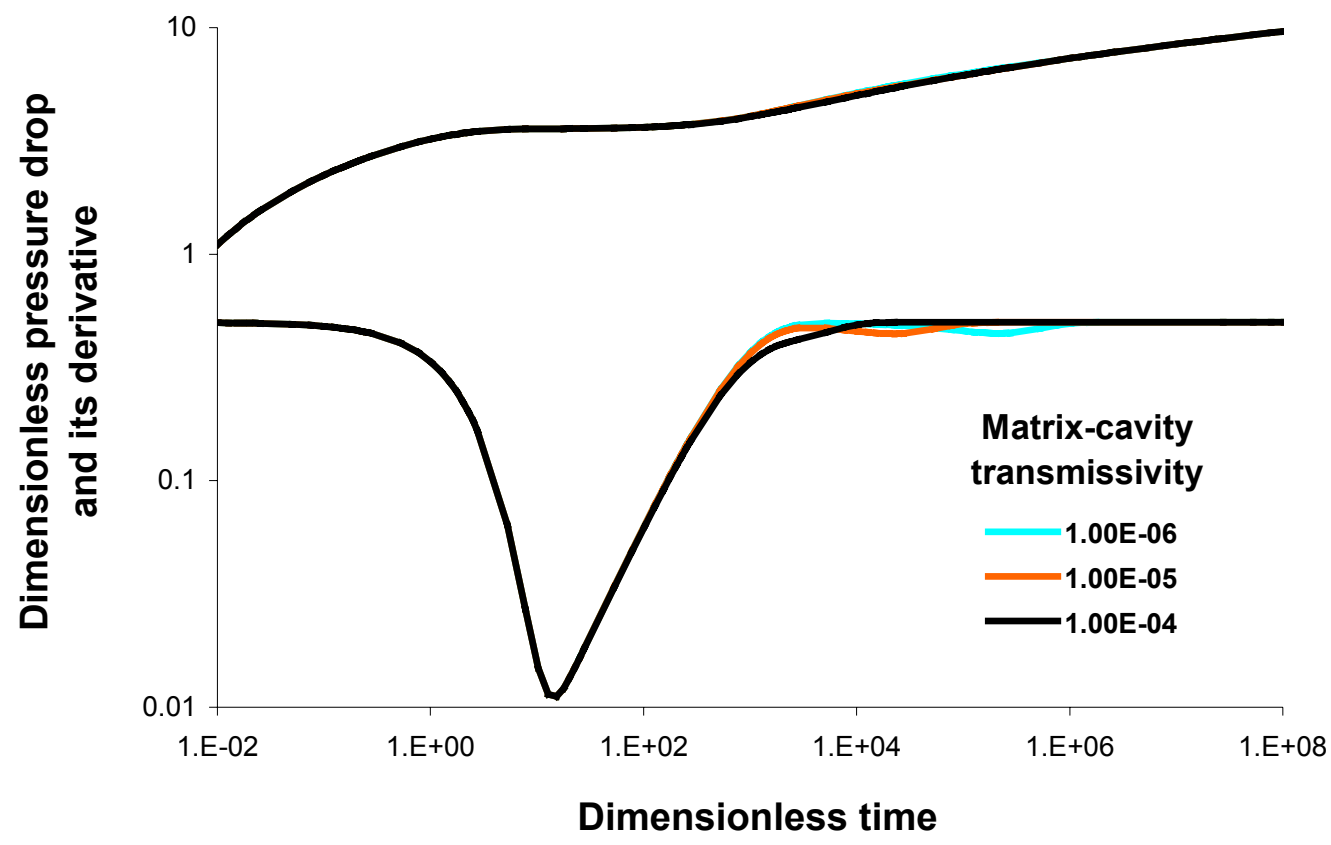

Fig. 8. Sensitivity of matrix-cavity transmissivity ratio. 


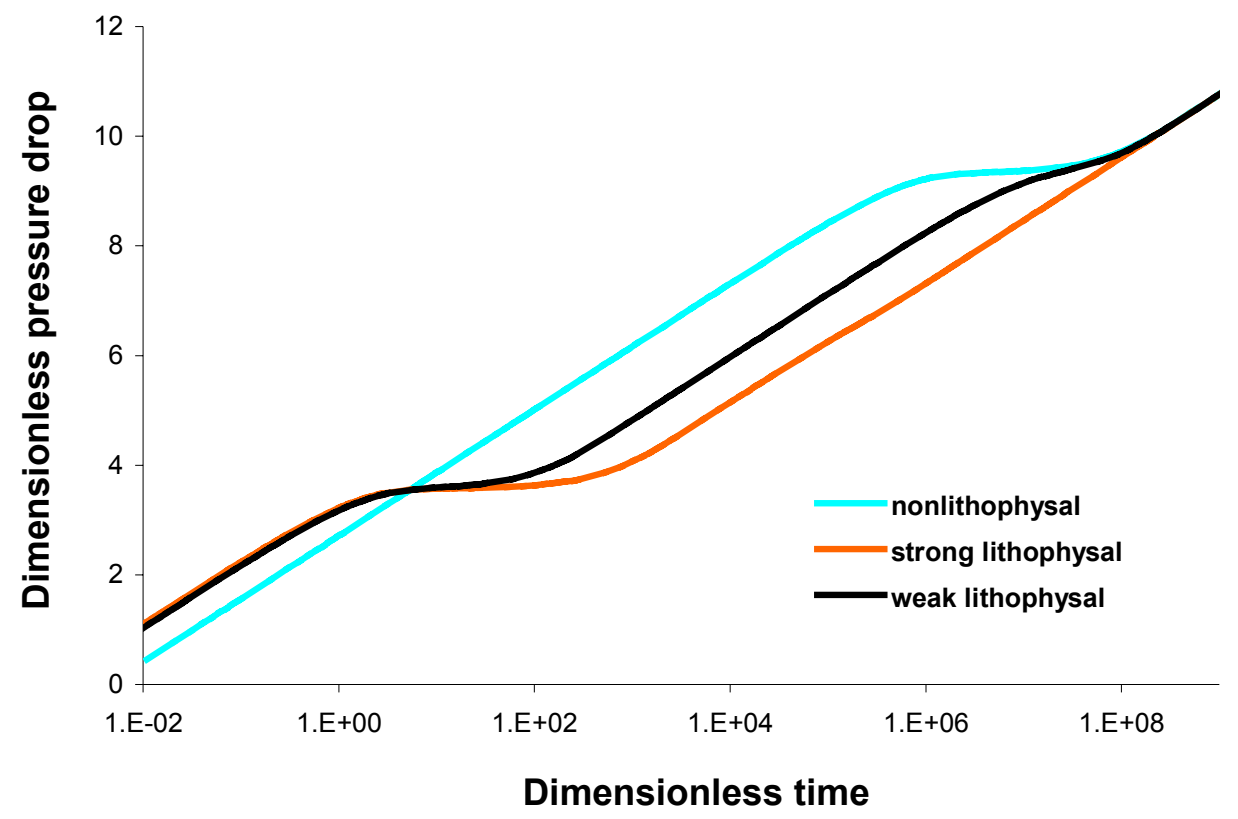

Fig. 9. Sensitivity of lithophysal volume displayed in pressure drawdown curve.

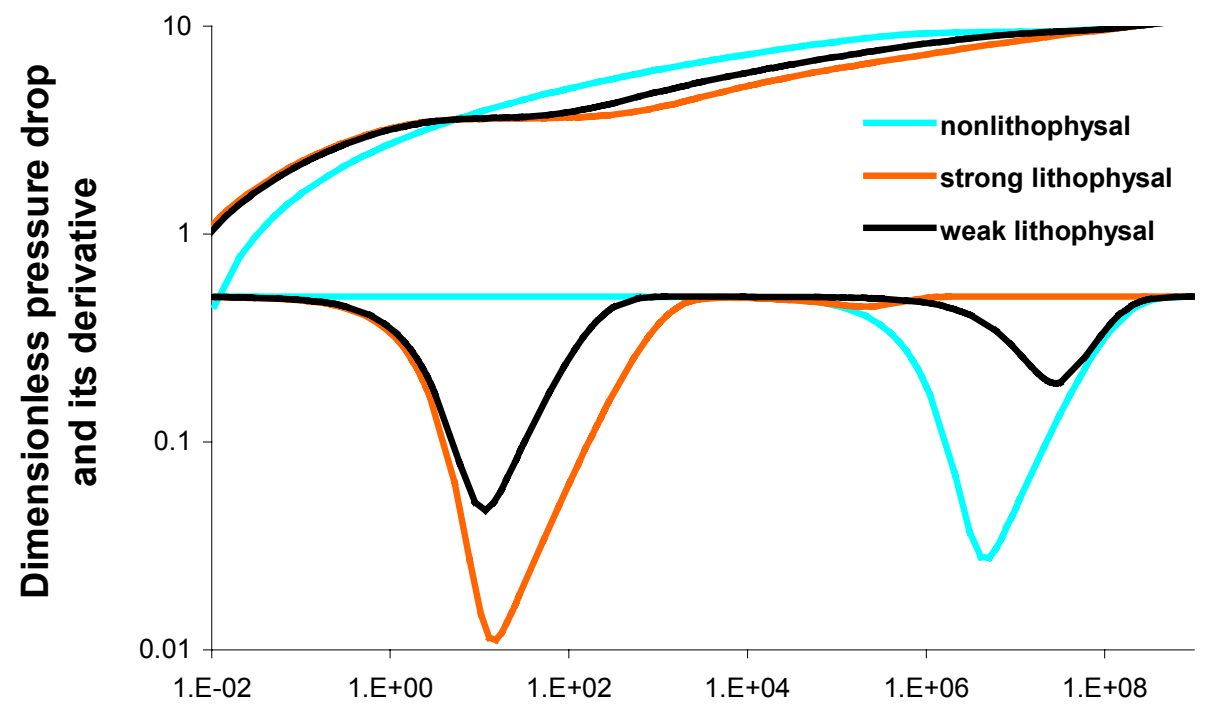

\section{Dimensionless time}

Fig. 10. Sensitivity of lithophysal volume displayed in pressure derivative plot. 


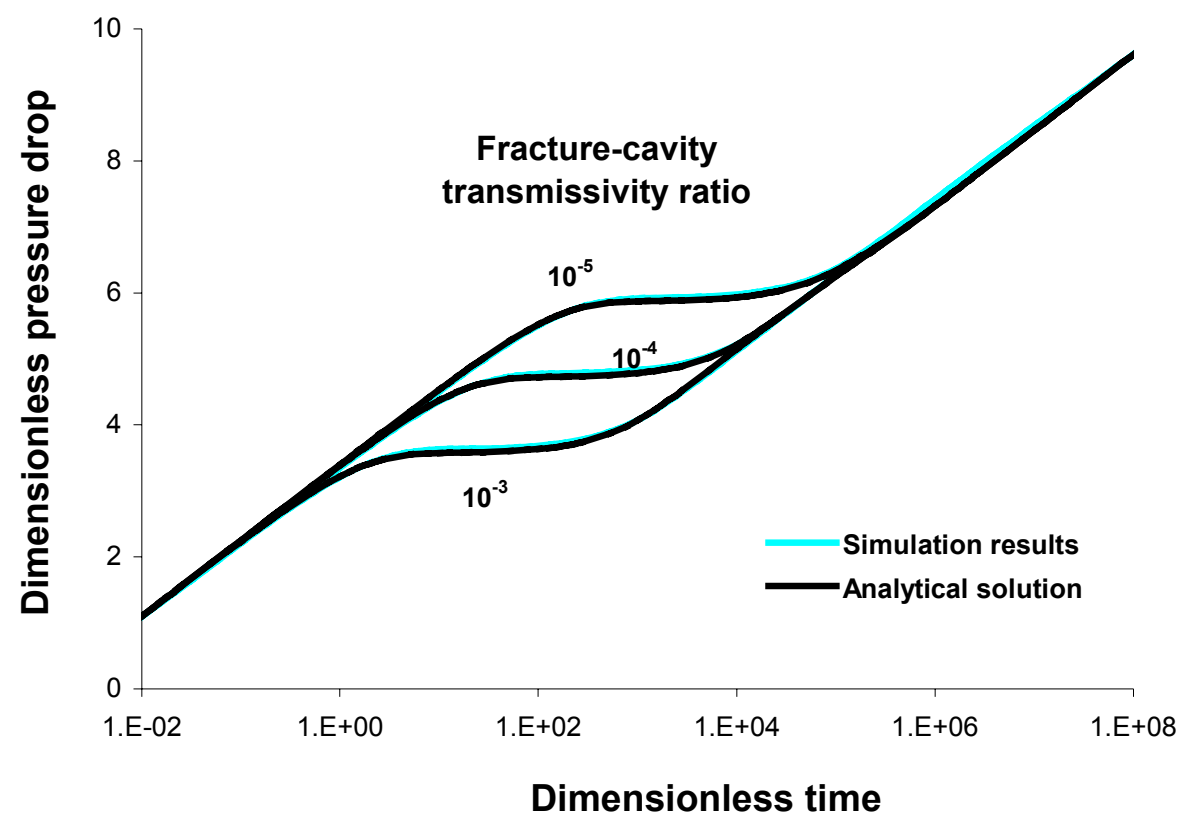

Fig. 11. Comparison of simulated pressure drawdown curves with analytical solutions.

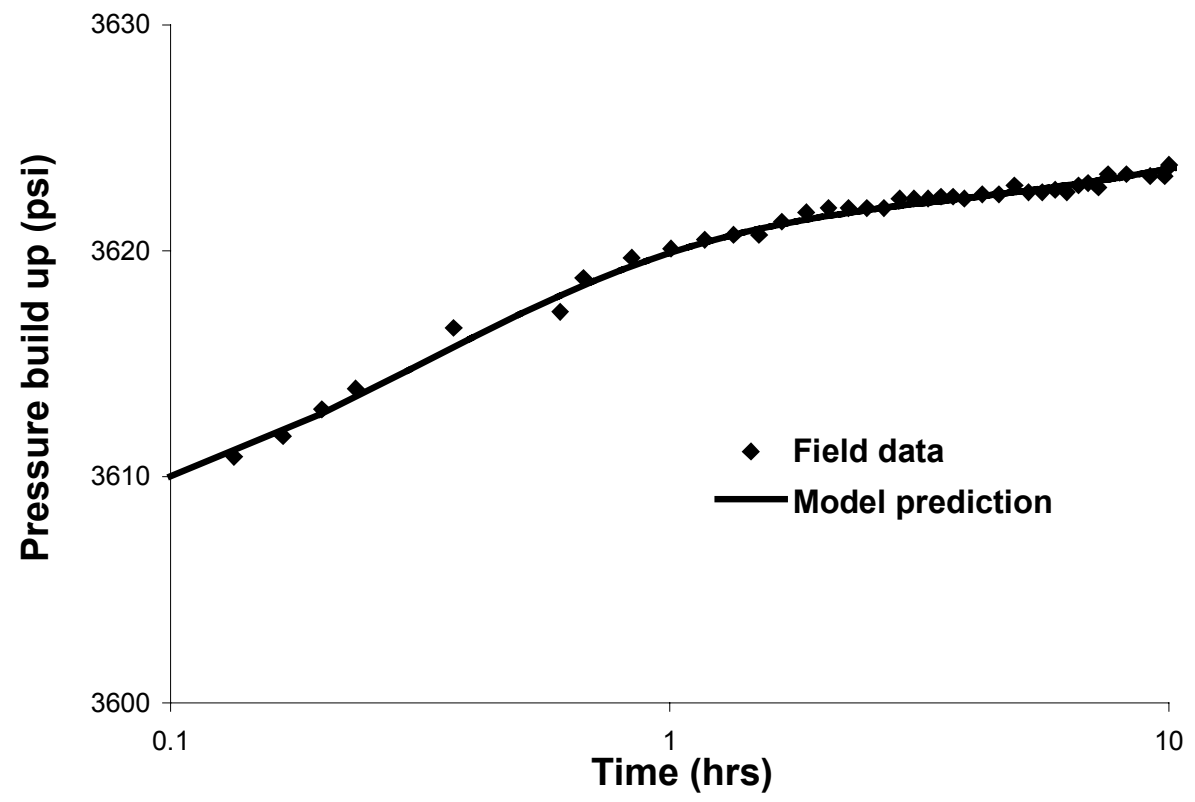

Fig. 12. Comparison of model predictions with pressure buildup test data from Crawford et al, 1976. 


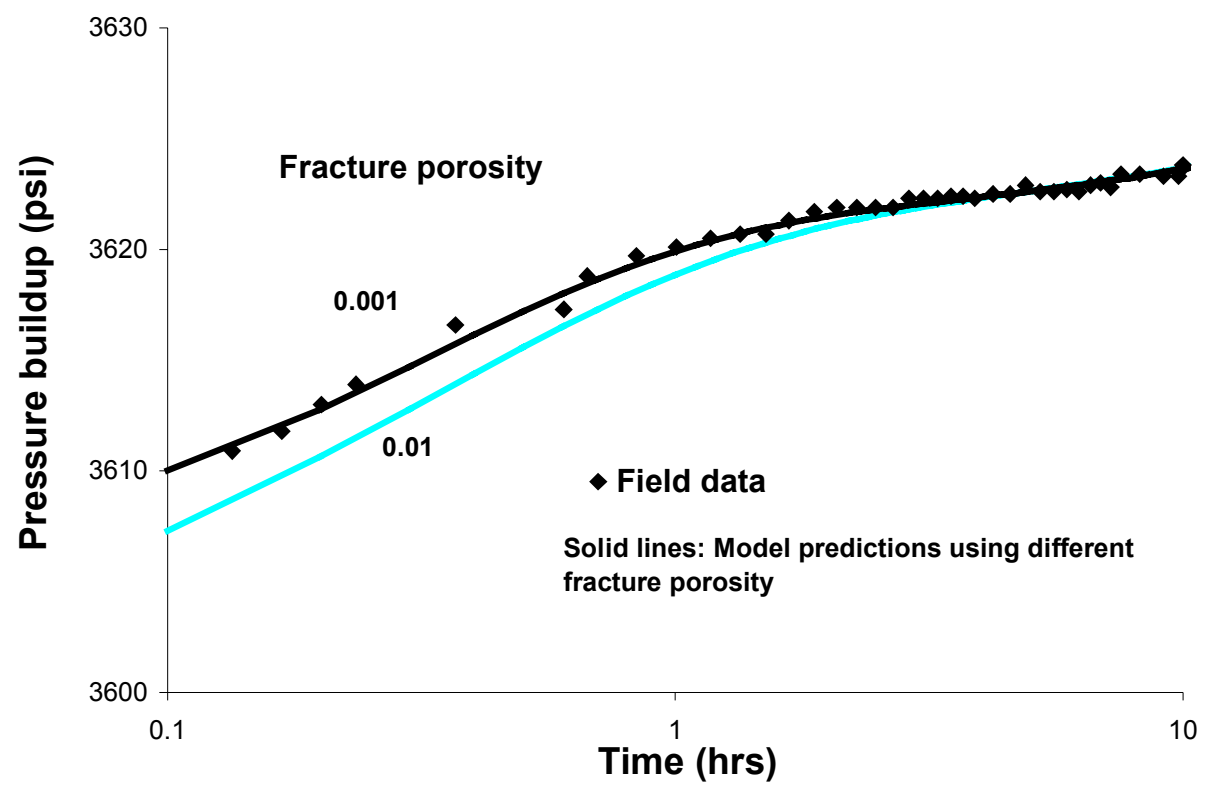

Fig. 13. Sensitivity of fracture porosity to the predicted pressure buildup in the field test case (Crawford et al., 1976).

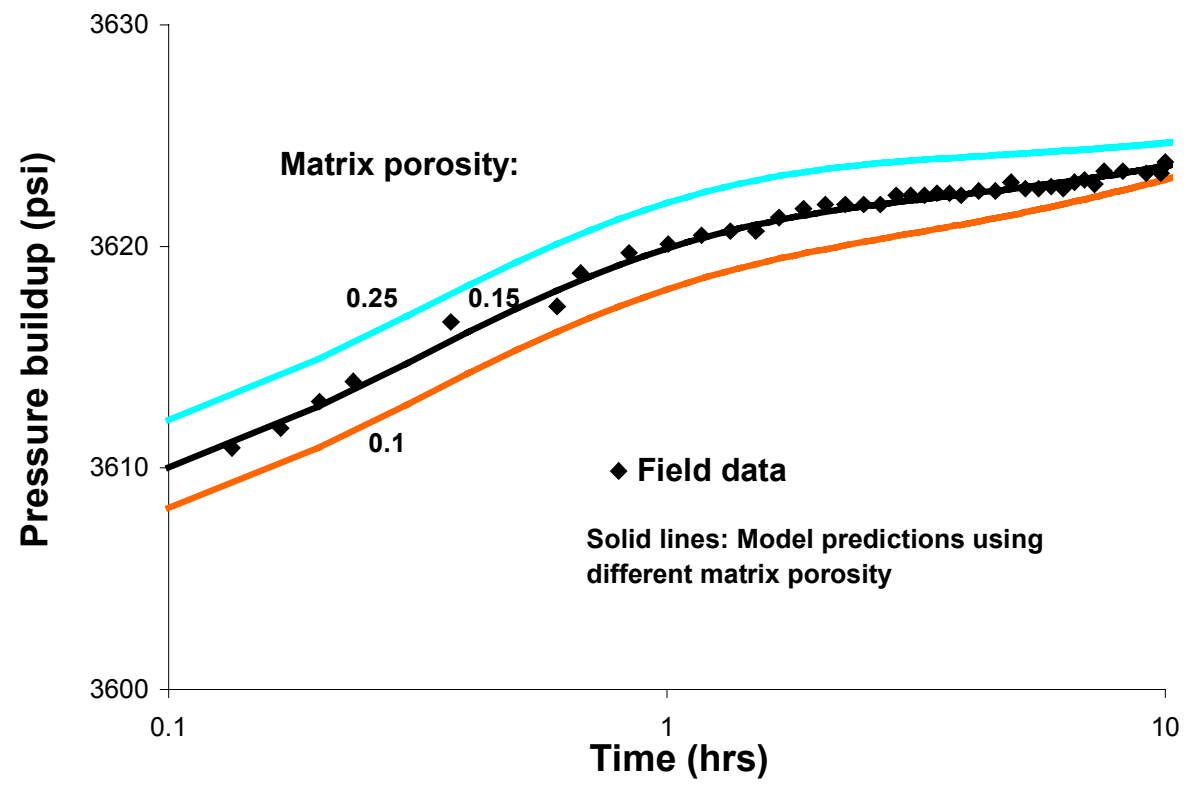

Fig. 14. Sensitivity of matrix porosity to the predicted pressure buildup in the field test case (Crawford et al., 1976). 


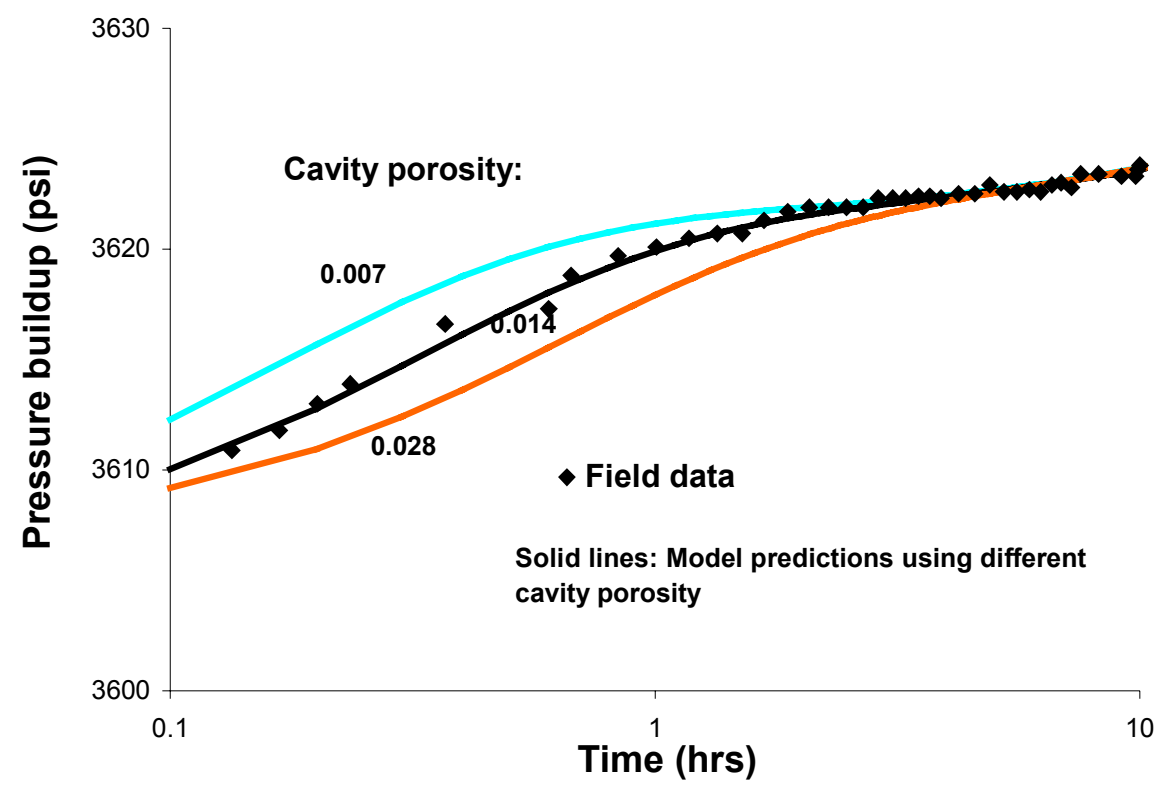

Fig. 15. Sensitivity of cavity porosity to the predicted pressure buildup in the field test case (Crawford et al., 1976).

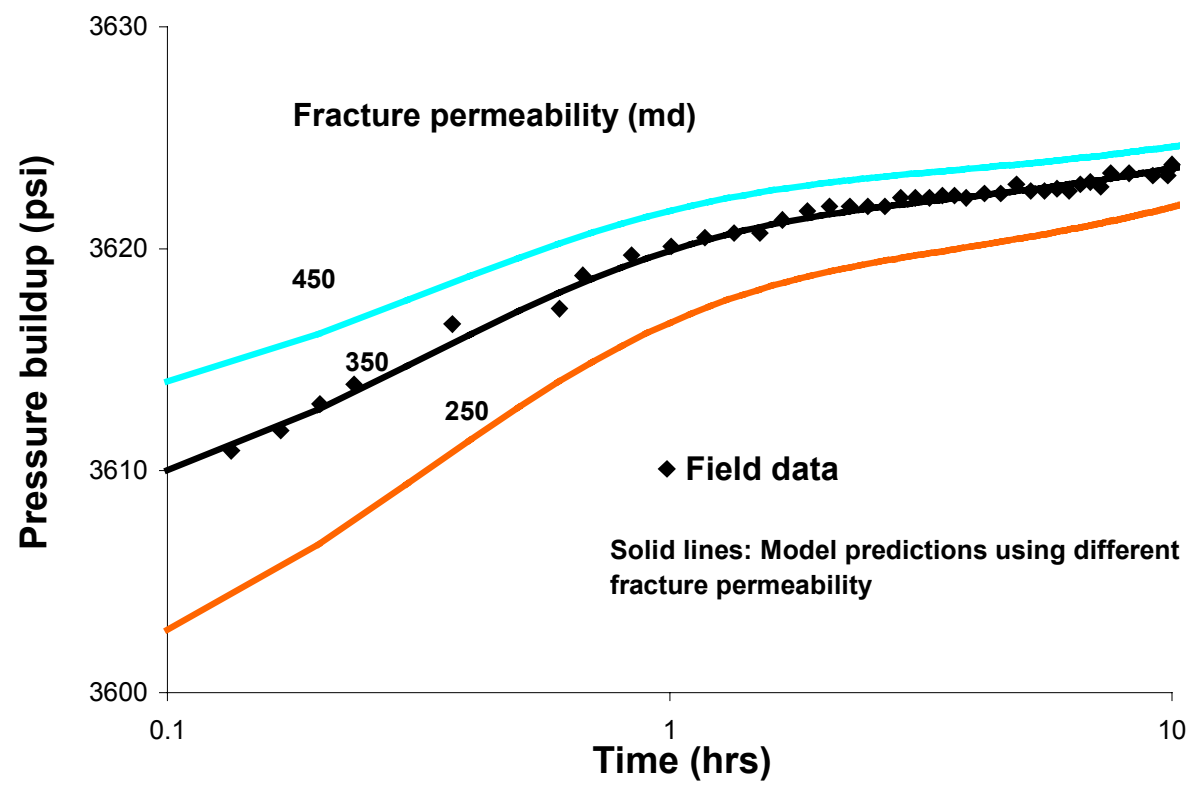

Fig. 16. Sensitivity of fracture permeability to the predicted pressure buildup in the field test case (Crawford et al., 1976). 


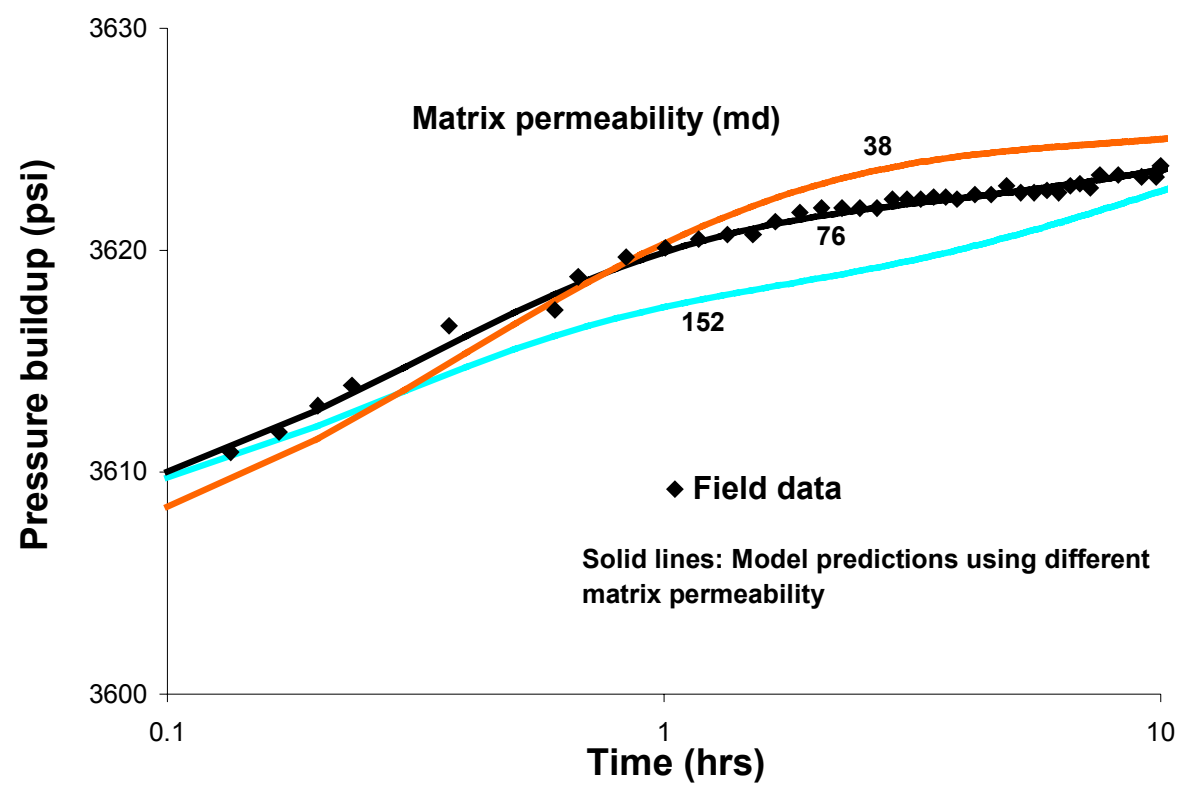

Fig. 17. Sensitivity of matrix permeability to the predicted pressure buildup in the field test case (Crawford et al., 1976). 